\title{
Design Concept for the In Situ Nanoprobe Beamline for the APS Upgrade
}

Jörg Maser $^{1,}{ }^{*}$, Barry Lai $^{1}$, Vincent De Andrade ${ }^{1}$, Simon R. Bare ${ }^{2}$, Mariana Bertoni $^{3}$, Tonio Buonassisi ${ }^{4}$, Paul Evans ${ }^{5}$, David P. Fenning ${ }^{6}$, Steve Heald ${ }^{1}$, Chris Johnson ${ }^{7}$, Tony Lanzirotti ${ }^{8}$, Conal Murray ${ }^{9}$, Tijana Rajh $^{10}$, Volker Rose ${ }^{1,10}$, Ruben Reininger ${ }^{1}, X_{1}$ ianbo Shi ${ }^{1}$, M. Stuckelberger ${ }^{11}$, David Tiede ${ }^{7}$, Stefan Vogt ${ }^{1}$, Randy Winans ${ }^{1}$.

1. X-ray Science Division, Advanced Photon Source, Argonne National Laboratory, Argonne, IL, USA

2. SSRL, SLAC National Accelerator Laboratory, CA, USA.

3. Arizona State University, Tempe, AZ, USA.

4. Massachusetts Institute of Technology, Cambridge, MA, USA.

5. University of Wisconsin-Madison, WI, USA

6. University of California, San Diego, USA.

7. Chemical Sciences and Engineering Division, Argonne, IL, USA.

8. The University of Chicago, IL, USA.

9. IBM T.J. Watson Research Center, Yorktown Heights, NY, USA.

${ }^{10}$ Center for Nanoscale Materials, Argonne National Laboratory, Argonne, IL, USA

${ }^{11}$ Deutsches Elektronen-Synchrotron, Hamburg, Germany

* Corresponding author, maser@anl.gov

The Upgrade of the Advanced Photon Source with an Multibend Achromat (MBA) magnetic lattice, APS$\mathrm{U}$, will provide massively increased coherent $\mathrm{x}$-ray flux in the hard $\mathrm{x}$-ray range, and a factor of 20 decrease in the horizontal x-ray source size. The upgrade creates an x-ray source that is ideally suited for applications requiring diffraction-limited focusing, such as X-ray nanoprobes, and coherent techniques such as ptychography. To study advanced materials during fabrication and operation, the APS Upgrade will build a next-generation hard x-ray nanoprobe beamline, the In-Situ Nanoprobe (ISN) beamline [1]. The ISN beamline will provide an achromatic focal spot of $20 \mathrm{~nm}$ and a working distance of $50 \mathrm{~mm}$ by using Kirkpatrick-Baez (K-B) mirrors in diffraction-limited configuration as nanofocusing optics. The large working distance enables broad in-situ environments, including heating, cooling, flow of process gases and fluids, and application of electric fields. The ISN beamline will provide a range of photon energies between $4.8 \mathrm{keV}$ and $30 \mathrm{keV}$, providing access to $\mathrm{K}$ or L absorption edges of most elements in the periodic table. The ISN instrument is designed as scanning nanoprobe, with $\mathrm{x}$-ray fluorescence detection and ptychography as major contrast modes. A secondary area detector will collect diffracted xrays and provide limited capability in identifying the local crystalline state.

Figure 1 shows the optical concept of the ISN beamline. The key optical aspect of the design is a set of vertical and horizontal spatial filters (SFs). Each SF consists of a focusing mirror and a beam-defining aperture (BDA). The first beamline optic is a flat, vertically deflecting high-heatload mirror at $28 \mathrm{~m}$ from the source, followed by a vertically focusing pink-beam mirror at $29 \mathrm{~m}$. A horizontally deflecting crystal monochromator $(\mathrm{DCM})$ with a bandpass $\triangle \mathrm{E} / \mathrm{E}$ of $10^{-4}$ for spectroscopy, and a double multilayer monochromator (DMM) with $\Delta \mathrm{E} / \mathrm{E}$ of $10^{-2}$ are placed at $30 \mathrm{~m}$ and at $32 \mathrm{~m}$, respectively. A horizontally focusing mirror is positioned at $43 \mathrm{~m}$, and only accepts monochromatic beam. This setup provides a clean secondary source in the horizontal and vertical direction. The vertically aperturing BDA is placed at 55 $\mathrm{m}$, the horizontally aperturing BDA at $64 \mathrm{~m}$, to provide the lateral coherence lengths required to coherently illuminate the horizontally focusing and vertically focusing K-B mirrors. All beamline optics are positioned upstream of the BDA, and therefore allow to remove the effects of source motion, optics motion or wavefront deterioration from affecting the position or shape of the focused beam in the ISN instrument. 
This positioning of the beamline optics exposes the BDAs to only focused monochromatic beam, without engineering constraints imposed by high heatload design. The BDAs are thus highly stable and can be moved with high accuracy and reproducibility. Adjustment of the secondary source size by opening or closing the BDA allows the manipulation of the lateral coherence length at the ISN position.

The ISN instrument will be positioned at a distance of $220 \mathrm{~m}$ from the source. The long propagation distance from the BDA's to the ISN instrument provides a lateral coherence length sufficiently large to match the acceptance of the nanofocusing K-B mirrors, $305 \mu \mathrm{m}(\mathrm{h}) \times 1 \mathrm{~mm}(\mathrm{v})$, and thereby enables a large working distance of more than $50 \mathrm{~mm}$. To accept the incoming coherent beam, a vertically focusing mirror with a length of $400 \mathrm{~mm}$ and a horizontally focusing mirror with a length of $122 \mathrm{~mm}$ will be used. While even short mirrors with the required small slope errors could not be fabricated in the past, the development of advanced machining approaches now makes long, diffraction-limited mirrors feasible. For positioning such mirrors, flexure-based positioners with nano-radian angular resolution, as show in Fig. 2, will be used [3].

The long working distance and small spot size, coupled with high coherent flux, will enable the ISN to study hierarchical materials and devices, such as photovoltaic systems [4], battery materials and nanoelectronic devices, with high elemental sensitivity, under a broad range of in-situ conditions. That will allow, for example, the study of evolution of defects during materials fabrication and device operation, and give insight into defect engineering of advanced materials and materials systems.

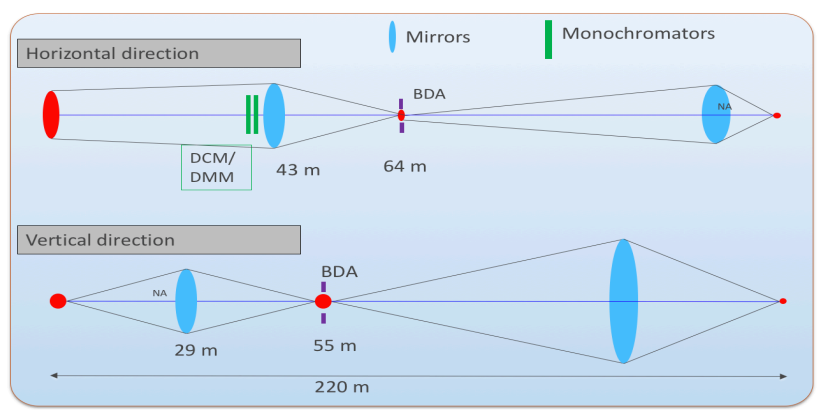

Figure. 1. Optical layout of the ISN beamline. The beamline is designed to accommodate a highly astigmatic K-B nanofocusing optic with length of $122 \mathrm{~mm}(\mathrm{~h}) \times 400 \mathrm{~mm}(\mathrm{v})$. The beamline is therefore astigmatic as well, with the secondary source for the horizontal direction positioned at 64 $\mathrm{m}$, and for the vertical direction at $55 \mathrm{~m}$.
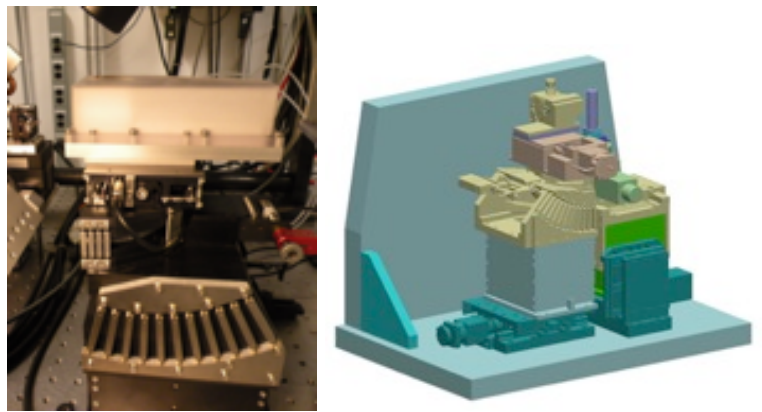

Figure. 2. Flexure-based nanopositioner for K-B mirror system [3].

Each nanofocusing mirror must be positioned with an angular accuracy of $10 \mathrm{n}$-rad, and positional stability of better than $5 \mathrm{~nm}$. Composite flexures positioned along an arc allow rotation of each mirror around an axis through the mirror surface.

\section{References:}

[1] J. Maser et al., Metall. Mater. Trans. DOI: 10.1007/s11661-013-1901-x, (2013).

[2] H. Mimura, S. Handa, T. Kimura et al, Nat. Phys. (2010)

doi:10.1038/nphys 14

[3] D. Shu et al., Proceedings of 28th ASPE Annual Meeting, (2013)

[4] Bertoni M. Stuckelberger, T. Nietzold, et al., J-PV, (2017), doi: 10.1109/JPHOTOV.2017.2762584.

[5] This research used resources of the Advanced Photon Source, a U.S. Department of Energy (DOE) Office of Science User Facility operated for the DOE Office of Science by Argonne National Laboratory under Contract No. DE-AC02-06CH11357. 